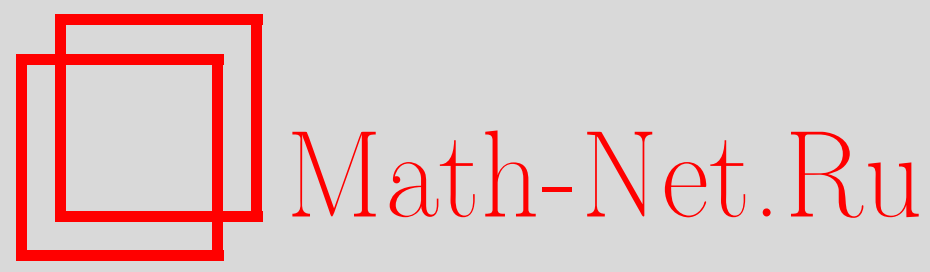

В. А. Кирушев, В. Н. Малоземов, А. Б. Певный, Вейвлетное разложение пространства дискретных периодических сплайнов, Матем. заметки, 2000, том 67, выпуск 5, 712720

DOI: https://doi.org/10.4213/mzm888

Использование Общероссийского математического портала Math-Net.Ru подразумевает, что вы прочитали и согласны с пользовательским соглашением http://www.mathnet.ru/rus/agreement

Параметры загрузки:

IP: 52.90 .164 .192

26 апреля 2023 г., 18:05:32

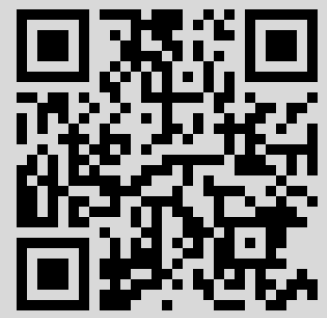




\title{
ВЕЙВЛЕТНОЕ РАЗЛОЖЕНИЕ
}

\section{ПРОСТРАНСТВА ДИСКРЕТНЫХ ПЕРИОДИЧЕСКИХ СПЛАЙНОВ}

\author{
В. А. Кирушев, В. Н. Малоземов, А.Б. Певный
}

В пространстве $\mathscr{S}_{r}^{m}$ дискретных периодических сплайнов построен ортогональный базис. С его помощью при $m=2^{t}$ получено вейвлетное разложение пространства $\mathscr{S}_{r}^{m}$. Выведены рекуррентные формулы для перехода от разложения по ортогональному базису к вейвлетному разложению и рекуррентные формулы для обратного перехода.

Библиографояи: 5 названий.

1. Пусть $m, n, r-$ натуральные числа и $N=m n$. Положим

$$
u(k)= \begin{cases}n^{2} & \text { при } k=0, \\ \left(\frac{\sin (\pi k / m)}{\sin (\pi k / N)}\right)^{2} & \text { при } k=1, \ldots, N-1 .\end{cases}
$$

Функция целочисленного аргумента

$$
Q_{r}(j)=\frac{1}{N} \sum_{k=0}^{N-1} u^{r}(k) \omega_{N}^{j k}, \quad j \in \mathbb{Z},
$$

где $\omega_{N}=\exp (2 \pi i / N)$, называется дискретным периодическим B-сплайном порядка $r$ [1]. Дискретный периодический сплайн порядка $r$ определяется как линейная комбинация с комплексньми коэффициентами сдвигов $B$-сплайна

$$
S(j)=\sum_{p=0}^{m-1} c(p) Q_{r}(j-p n) .
$$

Множество дискретных периодических сплайнов порядка $r$ обозначим через $\mathscr{S}_{r}^{m}$. Очевидно, что $\mathscr{S}_{r}^{1}$ есть пространство комплексных констант, а $\mathscr{S}_{r}^{N}=\mathbb{C}_{N}$, где $\mathbb{C}_{N}-$ пространство комплекснозначных $N$-периодических последовательностей (сигналов). Индекс $m$ у $\mathscr{S}_{r}^{m}$ обозначает размерность этого линейного пространства.

В данной заметке в пространстве $\mathscr{S}_{r}^{m}$ построен ортогональный базис. С его помощью при $m=2^{t}$ получено вейвлетное разложение пространства $\mathscr{S}_{r}^{m}$. Выведены рекуррентные формулы для перехода от разложения по ортогональному базису к вейвлетному разложению и рекуррентные формулы для обратного перехода.

Работа выполнена при финансовой поддержке Российского фонда фундаментальных исследований, грант № 96-01-00269. 
2. Положим

$$
\mu_{k}(j)=\frac{1}{m} \sum_{p=0}^{m-1} Q_{r}(j-p n) \omega_{m}^{k p}, \quad k=0,1, \ldots, m-1
$$

Лемма 1. Справедлива формула

$$
\mu_{k}(j)=\frac{1}{N} \sum_{l=0}^{n-1} u^{r}(l m+k) \omega_{N}^{j(l m+k)}, \quad k=0,1, \ldots, m-1
$$

ДокАЗАТЕЛЬСТВо. Согласно (1)

$$
\mu_{k}(j)=\frac{1}{m N} \sum_{p=0}^{m-1}\left(\sum_{s=0}^{N-1} u^{r}(s) \omega_{N}^{(j-p n) s}\right) \omega_{m}^{k p}=\frac{1}{N} \sum_{s=0}^{N-1} u^{r}(s) \omega_{N}^{j s}\left(\frac{1}{m} \sum_{p=0}^{m-1} \omega_{m}^{p(k-s)}\right)
$$

Воспользуемся формулой

$$
\frac{1}{m} \sum_{p=0}^{m-1} \omega_{m}^{j p}=\delta_{m}(j)
$$

где $\delta_{m}(j)$ - единичный $m$-периодичный импульс, равный единице при $j=0$ и нулю при $j=1, \ldots, m-1$. Получим

$$
\mu_{k}(j)=\frac{1}{N} \sum_{s=0}^{N-1} \delta_{m}(k-s) u^{r}(s) \omega_{N}^{j s}=\frac{1}{N} \sum_{l=0}^{n-1} u^{r}(l m+k) \omega_{N}^{j(l m+k)} .
$$

Лемма доказана.

Согласно определению $u(k)$ имеем $u(m)=u(2 m)=\cdots=u((n-1) m)=0$. Отсюда и из леммы 1 следует, что

$$
\mu_{0}(j)=\frac{1}{N} \sum_{l=0}^{n-1} u^{r}(l m) \omega_{n}^{j l} \equiv \frac{1}{N} n^{2 r}
$$

Введем в $\mathbb{C}_{N}$ скалярное произведение

$$
\langle x, y\rangle=\sum_{j=0}^{N-1} x(j) \overline{y(j)}
$$

и норму $\|x\|=\langle x, x\rangle^{1 / 2}$.

ТЕОрема 1. Сигналь $\left\{\mu_{k}(j)\right\}_{k=0}^{m-1}$ образуют ортогональный базис в пространcmвe $\mathscr{S}_{r}^{m}$. 
ДокАЗАТЕЛЬСТво. Очевидно, что все $\mu_{k}$ принадлежат $\mathscr{S}_{r}^{m}$. Проверим их ортогональность. При $k \neq s$ имеем в силу (3) и (4)

$$
\left\langle\mu_{k}, \mu_{s}\right\rangle=\frac{1}{N} \sum_{p, q=0}^{n-1} u^{r}(p m+k) u^{r}(q m+s) \delta_{N}(p m+k-q m-s) .
$$

Поскольку $k \neq s$ и $k, s=0,1, \ldots, m-1$, последовательности индексов $\{p m+k\}$ и $\{q m+s\}$ не имеют общих точек и не выходят из множества $\{0,1, \ldots, N-1\}$. Значит, правая часть в последней формуле равна нулю. Ортогональность системы $\left\{\mu_{k}\right\}_{k=0}^{m-1}$ установлена.

Остается учесть, что количество функций $\mu_{k}$ совпадает с размерностью пространства $\mathscr{S}_{r}^{m}$. Теорема доказана.

Отметим, что

$$
\left\|\mu_{k}\right\|^{2}=\frac{1}{N} \sum_{l=0}^{n-1} u^{2 r}(l m+k) .
$$

ЛЕмма 2. При $k=1, \ldots, m-1$ справедливо равенство

$$
\mu_{m-k}(j)=\overline{\mu_{k}(j)}, \quad j \in \mathbb{Z}
$$

ДокАЗАТЕЛЬСТво. В силу определения $u(k)$ имеем $u(N-k)=u(k)$ при $k=1, \ldots$, $N-1$. Отсюда следует, что $B$-сплайн $Q_{r}(j)$ принимает только вещественные значения.

Далее,

$$
\overline{\mu_{m-k}(j)}=\frac{1}{m} \sum_{p=0}^{m-1} Q_{r}(j-p n) \omega_{m}^{-(m-k) p}=\mu_{k}(j) .
$$

Это равенство равносильно (5). Лемма доказана.

Сигнал $x \in \mathbb{C}_{N}$ называется четным м, если $x(N-j)=\overline{x(j)}$ при всех $j \in \mathbb{Z}$.

При фиксированном $j \in \mathbb{Z}$ продолжим $\mu_{k}(j)$ по индексу $k$ периодически с периодом $m$. Согласно лемме 2 последовательность $\left\{\mu_{k}(j)\right\}$ будет четной по $k$.

ЛЕмма 3. При всех иелых $k, l$ справедливо равенство

$$
\mu_{k}(j-l n)=\omega_{m}^{-k l} \mu_{k}(j), \quad j \in \mathbb{Z}
$$

ДокАЗАТЕЛьство. Действительно,

$$
\mu_{k}(j-l n)=\frac{1}{m} \sum_{p=0}^{m-1} Q_{r}(j-(l+p) n) \omega_{m}^{k(l+p)-k l}=\omega_{m}^{-k l} \mu_{k}(j) .
$$

ЛЕмма 4. Пусть $k \in\{1, \ldots, m-1\}$ и $s$-натуральное число. Если произведение ks не делится на $\mathrm{m}$, то

$$
\sum_{j=0}^{N-1}\left[\mu_{k}(j)\right]^{s}=0
$$


ДОКАЗАТЕЛЬСТВО основано на том, что согласно (6) и (4)

$$
\sum_{j=0}^{N-1}\left[\mu_{k}(j)\right]^{s}=\sum_{l=0}^{m-1} \sum_{q=0}^{n-1}\left[\mu_{k}(q+l n)\right]^{s}=\sum_{l=0}^{m-1} \omega_{m}^{l k s} \sum_{q=0}^{n-1}\left[\mu_{k}(q)\right]^{s}=m \delta_{m}(k s) \sum_{q=0}^{n-1}\left[\mu_{k}(q)\right]^{s} .
$$

В частности, при $k=1, \ldots, m-1, k \neq m / 2$, справедливо равенство

$$
\sum_{j=0}^{N-1}\left[\mu_{k}(j)\right]^{2}=0
$$

Отметим, что при четном $m$

$$
\mu_{m / 2}(j)=\frac{1}{m} \sum_{p=0}^{m-1}(-1)^{p} Q_{r}(j-p n)
$$

3. В дальнейшем считаем, что $m=2^{t}$. Положим $m_{\nu}=m / 2^{\nu}, n_{\nu}=2^{\nu} n$. В этом случае $m_{\nu} n_{\nu}=N$ при всех $\nu=0,1, \ldots, t$.

Обозначим через $\mu_{k}^{\nu}$ ортогональные сплайны, соответствуюшие параметрам $m_{\nu}, n_{\nu}$. В частности, $\mu_{k}^{0}=\mu_{k}$. Следующая лемма играет фундаментальную роль в вопросах декомпозиции пространства $\mathscr{S}_{r}^{m}$.

ЛЕмма 5. При $\nu=0,1, \ldots, t-1$ справедлива рекуррентная формула

$$
\mu_{k}^{\nu+1}(j)=c^{\nu}(k) \mu_{k}^{\nu}(j)+c^{\nu}\left(k+m_{\nu+1}\right) \mu_{k+m_{\nu+1}}^{\nu}(j)
$$

əде $c^{\nu}(k)=\left(2 \cos \pi k / m_{\nu}\right)^{2 r}$.

ДокАЗАТЕЛЬСТво. При $k=0$ формула $(7)$ принимает вид $\mu_{0}^{\nu+1}(j)=2^{2 r} \mu_{0}^{\nu}(j)$. Она верна, поскольку $\mu_{0}^{\nu}(j) \equiv n_{\nu}^{2 r} / N$.

Пусть $k=1, \ldots, m_{\nu+1}-1$. Тогда

$$
\begin{aligned}
\mu_{k}^{\nu+1}(j) & =\frac{1}{N} \sum_{l=0}^{n_{\nu+1}-1} u^{r}\left(l m_{\nu+1}+k\right) \omega_{N}^{j\left(l m_{\nu+1}+k\right)} \\
& =\frac{1}{N}\left(\sin \frac{\pi k}{m_{\nu+1}}\right)^{2 r} \sum_{l=0}^{2 n_{\nu}-1}\left(\sin \frac{\pi\left(l m_{\nu+1}+k\right)}{N}\right)^{-2 r} \omega_{N}^{j\left(l m_{\nu+1}+k\right)}
\end{aligned}
$$

Последнюю сумму разобьем на две суммы: по четным индексам $l=2 s$ и по нечетньп $l=2 s+1, s=0,1, \ldots, n_{\nu}-1$. Получим

$$
\begin{aligned}
\mu_{k}^{\nu+1}(j)= & \frac{1}{N}\left(\sin \frac{2 \pi k}{m_{\nu}}\right)^{2 r} \sum_{s=0}^{n_{\nu}-1}\left(\sin \frac{\pi\left(s m_{\nu}+k\right)}{N}\right)^{-2 r} \omega_{N}^{j\left(s m_{\nu}+k\right)} \\
& +\frac{1}{N}\left(\sin \frac{2 \pi k}{m_{\nu}}\right)^{2 r} \sum_{s=0}^{n_{\nu}-1}\left(\sin \frac{\pi\left(s m_{\nu}+k+m_{\nu+1}\right)}{N}\right)^{-2 r} \omega_{N}^{j\left(s m_{\nu}+k+m_{\nu+1}\right)} .
\end{aligned}
$$

Так как $\sin \left(2 \pi k / m_{\nu}\right)=2 \cos \left(\pi k / m_{\nu}\right) \sin \left(\pi k / m_{\nu}\right)$ и

$$
\sin \frac{2 \pi k}{m_{\nu}}=-\sin \frac{2 \pi\left(k+m_{\nu+1}\right)}{m_{\nu}}=-2 \cos \frac{\pi\left(k+m_{\nu+1}\right)}{m_{\nu}} \sin \frac{\pi\left(k+m_{\nu+1}\right)}{m_{\nu}},
$$

то $\mu_{k}^{\nu+1}(j)=c^{\nu}(k) \mu_{k}^{\nu}(j)+c^{\nu}\left(k+m_{\nu+1}\right) \mu_{k+m_{\nu+1}}^{\nu}(j)$. Лемма доказана.

Из этой леммы, в частности, следует, что $\mathscr{S}_{r}^{m_{\nu+1}} \subset \mathscr{S}_{r}^{m_{\nu}}$ при $\nu=0,1, \ldots, t-1$. 
4. Введем дискретные сплайны

$$
w_{k}^{\nu+1}(j)=d^{\nu}(k) \mu_{k}^{\nu}(j)+d^{\nu}\left(k+m_{\nu+1}\right) \mu_{k+m_{\nu+1}}^{\nu}(j)
$$

где $d^{\nu}(k)=\omega_{m_{\nu}}^{k} c^{\nu}\left(k+m_{\nu+1}\right)\left\|\mu_{k+m_{\nu+1}}^{\nu}\right\|^{2}$. Очевидно, что $w_{k}^{\nu+1} \in \mathscr{S}_{r}^{m_{\nu}}$.

Лемма 6. Сплайны $\left\{w_{k}^{\nu+1}\right\}_{k=0}^{m_{\nu+1}-1}$ образуют ортогональную систему. При этом $\left\langle w_{k}^{\nu+1}, \mu_{s}^{\nu+1}\right\rangle=0$ nрu всех $k, s=0,1, \ldots, m_{\nu+1}-1 u$

$$
\left\|w_{k}^{\nu+1}\right\|=\left\|\mu_{k}^{\nu}\right\|\left\|\mu_{k+m_{\nu+1}}^{\nu}\right\|\left\|\mu_{k}^{\nu+1}\right\| .
$$

ДокаЗАтЕльСтво. Равенства $\left\langle w_{k}^{\nu+1}, w_{s}^{\nu+1}\right\rangle=0$ и $\left\langle w_{k}^{\nu+1}, \mu_{s}^{\nu+1}\right\rangle=0$ при $k \neq s$, $k, s=0,1, \ldots, m_{\nu+1}-1$, следуют из $(7),(8)$ и ортогональности системы $\left\{\mu_{k}^{\nu}\right\}_{k=0}^{m_{\nu}-1}$. Остается проверить, что $\left\langle w_{k}^{\nu+1}, \mu_{k}^{\nu+1}\right\rangle=0$. Имеем

$$
\begin{aligned}
\left\langle w_{k}^{\nu+1}, \mu_{k}^{\nu+1}\right\rangle & =d^{\nu}(k) c^{\nu}(k)\left\|\mu_{k}^{\nu}\right\|^{2}+d^{\nu}\left(k+m_{\nu+1}\right) c^{\nu}\left(k+m_{\nu+1}\right)\left\|\mu_{k+m_{\nu+1}}^{\nu}\right\|^{2} \\
& =c^{\nu}(k) c^{\nu}\left(k+m_{\nu+1}\right)\left\|\mu_{k}^{\nu}\right\|^{2}\left\|\mu_{k+m_{\nu+1}}^{\nu}\right\|^{2}\left(\omega_{m_{\nu}}^{k}+\omega_{m_{\nu}}^{k+m_{\nu+1}}\right)=0 .
\end{aligned}
$$

Вычисление $\left\|w_{k}^{\nu+1}\right\|^{2}$ проводится непосредственно:

$$
\begin{aligned}
\left\|w_{k}^{\nu+1}\right\|^{2} & =\left|d^{\nu}(k)\right|^{2}\left\|\mu_{k}^{\nu}\right\|^{2}+\left|d^{\nu}\left(k+m_{\nu+1}\right)\right|^{2}\left\|\mu_{k+m_{\nu+1}}^{\nu}\right\|^{2} \\
& =\left\|\mu_{k}^{\nu}\right\|^{2}\left\|\mu_{k+m_{\nu+1}}^{\nu}\right\|^{2}\left(\left[c^{\nu}\left(k+m_{\nu+1}\right)\right]^{2}\left\|\mu_{k+m_{\nu+1}}^{\nu}\right\|^{2}+\left[c^{\nu}(k)\right]^{2}\left\|\mu_{k}^{\nu}\right\|^{2}\right) \\
& =\left\|\mu_{k}^{\nu}\right\|^{2}\left\|\mu_{k+m_{\nu+1}}^{\nu}\right\|^{2}\left\|\mu_{k}^{\nu+1}\right\|^{2} .
\end{aligned}
$$

Последнее равенство равносильно (9). Лемма доказана.

Отметим, что $d^{\nu}(0)=0$ и $d^{\nu}\left(m_{\nu}-k\right)=\overline{d^{\nu}(k)}$. Таким образом, $m_{\nu}$-периодическая последовательность $\left\{d^{\nu}(k)\right\}$ является четной. Нетрудно также проверить, что при фиксированном $j \in \mathbb{Z} m_{\nu+1}$-периодическая по $k$ последовательность $\left\{w_{k}^{\nu+1}(j)\right\}$ будет четной. При этом $w_{0}^{\nu+1}(j)=-2^{2 r}\left\|\mu_{0}^{\nu}\right\|^{2} \mu_{m_{\nu+1}}^{\nu}(j)$.

Нам потребуется еще одно свойство, легко следующее из (6) и (8):

$w_{k}^{\nu+1}\left(j-\ln n_{\nu+1}\right)=d^{\nu}(k) \mu_{k}^{\nu}\left(j-2 \ln _{\nu}\right)+d^{\nu}\left(k+m_{\nu+1}\right) \mu_{k+m_{\nu+1}}^{\nu}\left(j-2 \ln n_{\nu}\right)=\omega_{m_{\nu+1}}^{-k l} w_{k}^{\nu+1}(j)$.

5. Обозначим через $\mathscr{W}_{r}^{m_{\nu+1}}$ линейную оболочку, натянутую на сплайны $w_{k}^{\nu+1}, k=0$, $1, \ldots, m_{\nu+1}-1$. Согласно лемме 6 пространство $\mathscr{S}_{r}^{m_{\nu}}$ можно рассматривать как ортогональную сумму подпространств $\mathscr{S}_{r}^{m_{\nu+1}}$ и $\mathscr{W}_{r}^{m_{\nu+1}}: \mathscr{S}_{r}^{m_{\nu}}=\mathscr{S}_{r}^{m_{\nu+1}} \oplus \mathscr{W}_{r}^{m_{\nu+1}}$.

Разложим $\mathscr{S}_{r}^{m}=\mathscr{S}_{r}^{m_{0}}$ по схеме

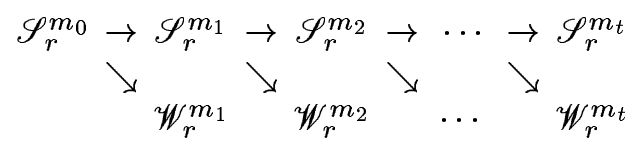

Придем к формуле

$$
\mathscr{S}_{r}^{m}=\mathscr{S}_{r}^{m_{t}} \oplus \mathscr{W}_{r}^{m_{t}} \oplus \mathscr{W}_{r}^{m_{t-1}} \oplus \cdots \oplus \mathscr{W}_{r}^{m_{1}}
$$

Здесь $\mathscr{S}_{r}^{m_{t}}=\mathscr{S}_{r}^{1}-$ одномерное комплексное пространство с образующей функцией $\mu_{0}^{t}(j) \equiv N^{2 r-1}$. 
6. Введем в $\mathscr{W}_{r}^{m_{\nu+1}}$ вейвлетньй базис. Для этого положим

$$
\theta_{r}^{\nu+1}(j)=\frac{1}{\sqrt{m_{\nu+1}}} \sum_{k=0}^{m_{\nu+1}-1} \frac{w_{k}^{\nu+1}(j)}{\left\|w_{k}^{\nu+1}\right\|} .
$$

Очевидно, что $\theta_{r}^{\nu+1} \in \mathscr{W}_{r}^{m_{\nu+1}}$.

ЛЕмма 7. Сдвиги $\theta_{r}^{\nu+1}\left(j-l n_{\nu+1}\right), l=0,1, \ldots, m_{\nu+1}-1$, образуют вещественный ортонормированный базис в $\mathscr{W}_{r}^{m_{\nu+1}}$.

ДоКАЗАТЕЛЬСТВо. Согласно (10)

$$
\theta_{r}^{\nu+1}\left(j-l n_{\nu+1}\right)=\frac{1}{\sqrt{m_{\nu+1}}} \sum_{k=0}^{m_{\nu+1}-1} \frac{\omega_{m_{\nu+1}}^{k l}}{\left\|w_{k}^{\nu+1}\right\|} w_{k}^{\nu+1}(j) .
$$

Далее,

$$
\left\langle\theta_{r}^{\nu+1}\left(\cdot-l n_{\nu+1}\right), \theta_{r}^{\nu+1}\left(\cdot-s n_{\nu+1}\right)\right\rangle=\frac{1}{m_{\nu+1}} \sum_{k=0}^{m_{\nu+1}-1} \omega_{m_{\nu+1}}^{k(s-l)}=\delta_{m_{\nu+1}}(s-l) .
$$

Тем самым установлено, что сплайны $\theta_{r}^{\nu+1}\left(j-l n_{\nu+1}\right), l=0,1, \ldots, m_{\nu+1}-1$, принадлежат подпространству $\mathscr{W}_{r}^{m_{\nu+1}}$ и образуют в нем ортонормированный базис.

Покажем, что функция $\theta_{r}^{\nu+1}(j)$ принимает только вешественные значения. Для этого перепишем формулу (12) с учетом (8) в виде

$$
\theta_{r}^{\nu+1}(j)=\frac{1}{\sqrt{m_{\nu+1}}} \sum_{k=0}^{m_{\nu}-1} \frac{d^{\nu}(k)}{\left\|w_{k}^{\nu+1}\right\|} \mu_{k}^{\nu}(j) .
$$

Обозначим $\sigma^{\nu}(k)=d^{\nu}(k) /\left(\sqrt{m_{\nu+1}}\left\|w_{k}^{\nu+1}\right\|\right)$. Отметим, что последовательность $\left\{\sigma^{\nu}(k)\right\}$ является четной. По определению

$$
\mu_{k}^{\nu}(j)=\frac{1}{m_{\nu}} \sum_{p=0}^{m_{\nu}-1} Q_{r}^{\nu}\left(j-p n_{\nu}\right) \omega_{m_{\nu}}^{k p}
$$

где $Q_{r}^{\nu}-B$-сплайн, соответствующий параметрам $m_{\nu}, n_{\nu}$. Подставив $(14)$ в (13), получим

$$
\theta_{r}^{\nu+1}(j)=\sum_{p=0}^{m_{\nu}-1} b^{\nu}(p) Q_{r}^{\nu}\left(j-p n_{\nu}\right) .
$$

Здесь $b^{\nu}(p)=m_{\nu}^{-1} \sum_{k=0}^{m_{\nu}-1} \sigma^{\nu}(k) \omega_{m_{\nu}}^{k p}$. Поскольку $\left\{\sigma^{\nu}(k)\right\}-$ четная последовательность, то коэффициенты $b^{\nu}(p)$ вещественны. $B$-сплайн $Q_{r}^{\nu}$ также принимает только вещественные значения. Значит, и вейвлет $\theta_{r}^{\nu+1}(j)$ принимает только вещественные значения. Лемма доказана.

Напомним, что $d^{\nu}(0)=0$, поэтому формулу (13) можно записать так:

$$
\theta_{r}^{\nu+1}(j)=\sum_{k=1}^{m_{\nu}-1} \sigma^{\nu}(k) \mu_{k}^{\nu}(j) .
$$

Отсюда, в частности, следует, что $\sum_{j=0}^{N-1} \theta_{r}^{\nu+1}(j)=0$.

Объединяя формулу (11) и лемму 7 , приходим к основному результату - вейвлетному разложению пространства $\mathscr{S}_{r}^{m}$. 
ТЕорема 2. При $m=2^{t}$ любой сплайн $S \in \mathscr{S}_{r}^{m}$ допускает представление

$$
S(j)=\alpha+\sum_{\nu=1}^{t} \sum_{l=0}^{m_{\nu}-1} \beta^{\nu}(l) \theta_{r}^{\nu}\left(j-\ln _{\nu}\right) .
$$

7. По-прежнему считаем, что $m=2^{t}$. Возьмем дискретньй периодический сплайн $S(j)$ вида (2) и положим

$$
\xi^{0}(k)=\sum_{p=0}^{m-1} c(p) \omega_{m}^{-k p}
$$

Получим

$$
S(j)=\sum_{k=0}^{m_{0}-1} \xi^{0}(k) \mu_{k}^{0}(j) .
$$

Вместе с тем, сплайн $S$ допускает представление

$$
S(j)=\sum_{k=0}^{m_{1}-1} \xi^{1}(k) \mu_{k}^{1}(j)+\sum_{l=0}^{m_{1}-1} \beta^{1}(l) \theta_{r}^{1}\left(j-l n_{1}\right) .
$$

Выведем формулы перехода от разложения (16) к разложению (17). Имеем

$$
\begin{aligned}
\xi^{1}(s)\left\|\mu_{s}^{1}\right\|^{2} & =\left\langle S, \mu_{s}^{1}\right\rangle=\left\langle\sum_{k=0}^{m_{0}-1} \xi^{0}(k) \mu_{k}^{0}, c^{0}(s) \mu_{s}^{0}+c^{0}\left(s+m_{1}\right) \mu_{s+m_{1}}^{0}\right\rangle \\
& =\xi^{0}(s) c^{0}(s)\left\|\mu_{s}^{0}\right\|^{2}+\xi^{0}\left(s+m_{1}\right) c^{0}\left(s+m_{1}\right)\left\|\mu_{s+m_{1}}^{0}\right\|^{2} .
\end{aligned}
$$

Отсюда следует, что

$$
\xi^{1}(k)=c^{0}(k) \frac{\left\|\mu_{k}^{0}\right\|^{2}}{\left\|\mu_{k}^{1}\right\|^{2}} \xi^{0}(k)+c^{0}\left(k+m_{1}\right) \frac{\left\|\mu_{k+m_{1}}^{0}\right\|^{2}}{\left\|\mu_{k}^{1}\right\|^{2}} \xi^{0}\left(k+m_{1}\right) .
$$

Далее,

$$
\begin{aligned}
\beta^{1}(s) & =\left\langle S, \theta_{r}^{1}\left(\cdot-s n_{1}\right)\right\rangle=\left\langle\sum_{k=0}^{m_{0}-1} \xi^{0}(k) \mu_{k}^{0}, \sum_{k=0}^{m_{0}-1} \frac{d^{0}(k) \omega_{m_{1}}^{-k s}}{\sqrt{m_{1}}\left\|w_{k}^{1}\right\|} \mu_{k}^{0}\right\rangle \\
& =\sum_{k=0}^{m_{0}-1} \frac{\overline{d^{0}(k)}\left\|\mu_{k}^{0}\right\|^{2}}{\sqrt{m_{1}}\left\|w_{k}^{1}\right\|} \xi^{0}(k) \omega_{m_{1}}^{k s} .
\end{aligned}
$$

Положим $\eta^{1}=\mathscr{F}_{m_{1}}\left(\beta^{1}\right)$, где $\mathscr{F}_{m_{1}}$ - дискретное преобразование Фурье порядка $m_{1}$. Компоненты сигнала $\eta^{1}$ вычисляются по формуле

$$
\begin{aligned}
\eta^{1}(l) & =\sum_{k=0}^{m_{0}-1} \frac{\overline{d^{0}(k)}\left\|\mu_{k}^{0}\right\|^{2}}{\sqrt{m_{1}}\left\|w_{k}^{1}\right\|} \xi^{0}(k) \sum_{s=0}^{m_{1}-1} \omega_{m_{1}}^{s(k-l)} \\
& =\sqrt{m_{1}} \sum_{k=0}^{m_{0}-1} \frac{\overline{d^{0}(k)}\left\|\mu_{k}^{0}\right\|^{2}}{\left\|w_{k}^{1}\right\|} \xi^{0}(k) \delta_{m_{1}}(k-l) \\
& =\sqrt{m_{1}} \frac{\left\|\mu_{l}^{0}\right\|\left\|\mu_{l+m_{1}}^{0}\right\|}{\left\|\mu_{l}^{1}\right\|}\left[c^{0}\left(l+m_{1}\right) \xi^{0}(l)-c^{0}(l) \xi^{0}\left(l+m_{1}\right)\right] \omega_{m_{0}}^{-l} .
\end{aligned}
$$


Мы воспользовались определением $d^{0}(k)$ и формулой $(9)$. Вектор коэффищиентов $\beta^{1}$ восстанавливается теперь по формуле обращения $\beta^{1}=\mathscr{F}_{m_{1}}^{-1}\left(\eta^{1}\right)$.

В общем случае для разложения сплайна $S \in \mathscr{S}_{r}^{m}$ по вейвлетному базису можно использовать следующую рекуррентную схему:

$$
\begin{aligned}
& \xi^{\nu+1}(k)=c^{\nu}(k) \frac{\left\|\mu_{k}^{\nu}\right\|^{2}}{\left\|\mu_{k}^{\nu+1}\right\|^{2}} \xi^{\nu}(k)+c^{\nu}\left(k+m_{\nu+1}\right) \frac{\left\|\mu_{k+m_{\nu+1}}^{\nu}\right\|^{2}}{\left\|\mu_{k}^{\nu+1}\right\|^{2}} \xi^{\nu}\left(k+m_{\nu+1}\right), \\
& \eta^{\nu+1}(k)=\sqrt{m_{\nu+1}} \frac{\left\|\mu_{k}^{\nu}\right\|\left\|\mu_{k+m_{\nu+1}}^{\nu}\right\|}{\left\|\mu_{k}^{\nu+1}\right\|}\left[c^{\nu}\left(k+m_{\nu+1}\right) \xi^{\nu}(k)-c^{\nu}(k) \xi^{\nu}\left(k+m_{\nu+1}\right)\right] \omega_{m_{\nu}}^{-k} .
\end{aligned}
$$

Здесь $k=0,1, \ldots, m_{\nu+1}-1, \nu=0,1, \ldots, t-1$. В результате придем к представлению (15), в котором $\alpha=\xi^{t}(0) \mu_{0}^{t}(j)=\xi^{t}(0) N^{2 r-1}$ и $\beta^{\nu}=\mathscr{F}_{m_{\nu}}^{-1}\left(\eta^{\nu}\right)$ при $\nu=1, \ldots, t$.

8. Рассмотрим обратную задачу перехода от представления (15) с $\alpha=\xi^{t}(0) N^{2 r-1}$ к представлению (16). Сделаем последний шаг преобразования. С учетом (7) и (13) имеем

$$
\begin{aligned}
\sum_{k=0}^{m_{1}-1} \xi^{1}(k) \mu_{k}^{1}(j) & =\sum_{k=0}^{m_{0}-1} \xi^{1}(k) c^{0}(k) \mu_{k}^{0}(j) \\
\sum_{l=0}^{m_{1}-1} \beta^{1}(l) \theta_{r}^{1}\left(j-l n_{1}\right) & =\sum_{l=0}^{m_{1}-1} \beta^{1}(l) \sum_{k=0}^{m_{0}-1} \frac{d^{0}(k) \omega_{m_{1}}^{-k l}}{\sqrt{m_{1}}\left\|w_{k}^{1}\right\|} \mu_{k}^{0}(j) \\
& =\sum_{k=0}^{m_{0}-1} \frac{d^{0}(k)}{\sqrt{m_{1}}\left\|w_{k}^{1}\right\|} \mu_{k}^{0}(j) \sum_{l=0}^{m_{1}-1} \beta^{1}(l) \omega_{m_{1}}^{-k l} \\
& =\sum_{k=0}^{m_{0}-1} \frac{\omega_{m_{0}}^{k} c^{0}\left(k+m_{1}\right)\left\|\mu_{k+m_{1}}^{0}\right\|}{\sqrt{m_{1}}\left\|\mu_{k}^{0}\right\|\left\|\mu_{k}^{1}\right\|} \eta^{1}(k) \mu_{k}^{0}(j),
\end{aligned}
$$

где $\eta^{1}=\mathscr{F}_{m_{1}}\left(\beta^{1}\right)$. Следовательно,

$$
S(j)=\sum_{k=0}^{m_{0}-1}\left[c^{0}(k) \xi^{1}(k)+\frac{\omega_{m_{0}}^{k} c^{0}\left(k+m_{1}\right)\left\|\mu_{k+m_{1}}^{0}\right\|}{\sqrt{m_{1}}\left\|\mu_{k}^{0}\right\|\left\|\mu_{k}^{1}\right\|} \eta^{1}(k)\right] \mu_{k}^{0}(j) .
$$

В общем случае преобразование коэффициентов вьполняется по формуле

$$
\xi^{\nu}(k)=c^{\nu}(k) \xi^{\nu+1}(k)+\frac{\omega_{m_{\nu}}^{k} c^{\nu}\left(k+m_{\nu+1}\right)\left\|\mu_{k+m_{\nu+1}}^{\nu}\right\|}{\sqrt{m_{\nu+1}}\left\|\mu_{k}^{\nu}\right\|\left\|\mu_{k}^{\nu+1}\right\|} \eta^{\nu+1}(k) .
$$

Здесь $k=0,1, \ldots, m_{\nu}-1, \nu=t-1, t-2, \ldots, 0$. Считаем, что последовательности с индексом $\nu+1$ продолжены периодически с периодом $m_{\nu+1}$.

9. На рис. 1 представлены графики вейвлетов $\theta_{2}^{5}(j), \theta_{2}^{4}(j), \ldots, \theta_{2}^{1}(j)$ при $m=32=2^{5}$ и $n=3$.

10. В непрерьвном случае аналогичные вопросы рассматривались в работах [2]-[4]. Теория кратно-масштабного анализа пространства $\mathbb{C}_{N}$ построена в [5]. 

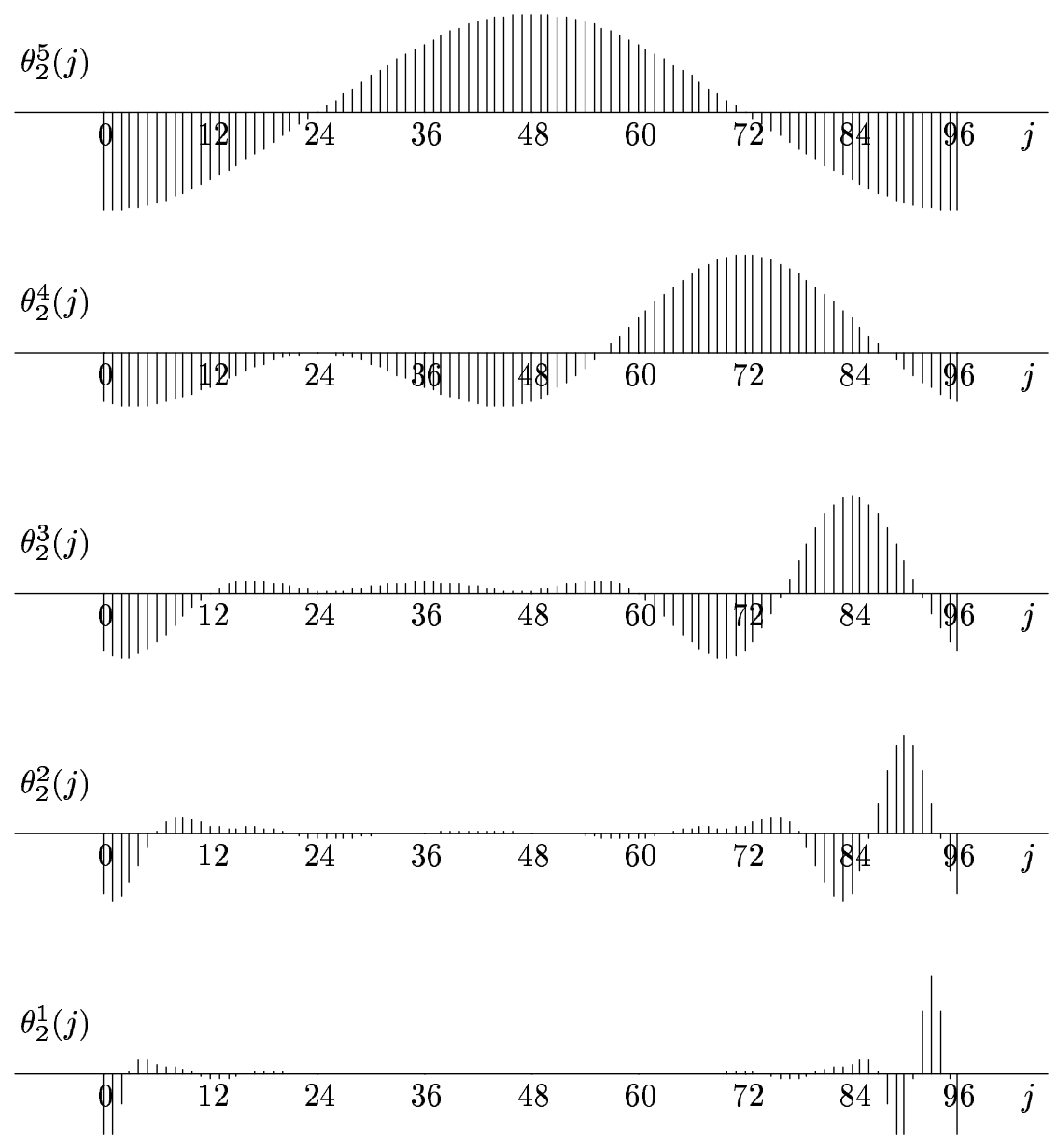

РИС. 1

\section{СПИСОК ЦИТИРОВАННОЙ ЛИТЕРАТУРЫ}

[1] Behr M. G., Malozemov V. N., Pevnyi A. B. A discrete version of spline-operational calculus. // International Conference on Optimization of Finite Element Approximations. Abstracts. St. Petersburg, 1995. P. 35.

[2] Kamada M., Toraichi K., Mori R. Periodic spline orthogonal bases // J. Approx. Theory. 1988. V. 55. № 1. Р. 27-34.

[3] ЖКелудев В.А. О вейвлетах на базе периодических сплайнов // Докл. РАН. 1994. Т. 335. №1. C. 9-13.

[4] Narcowich F. J., Ward J. D. Wavelets associated with periodic basis functions // Appl. Comput. Harmonic Anal. 1996. V. 3. № 1. P. 40-56.

[5] Петухов А. П. Периодические дискретные всплески // Алгебра и анализ. 1996. Т. 8. № 3. C. $151-183$.

Ськтывкарский государственный университет 